\title{
Access to the Labor Market of the Graduates of the Faculty of Law of the University of Barcelona (Spain)
}

\author{
Ana Antonia Collado ${ }^{1, *}$, Sandra Fachelli ${ }^{2}, \mathrm{Zi} \mathrm{Yu} \mathrm{Liu}{ }^{3}$ \\ ${ }^{1}$ Department of Sociology, Universidad de Barcelona, Barcelona, Spain \\ ${ }^{2}$ Department of Sociology, Universidad Autónoma de Barcelona, Bellaterra, Spain \\ ${ }^{3}$ Faculty of Law, Universidad de Barcelona, Barcelona, Spain \\ Email address: \\ antoniacollado@ub.edu (A. A. Collado), Sandra.Fachelli@uab.cat (S. Fachelli), liuziyu20@hotmail.com (Zi Yu Liu) \\ ${ }^{*}$ Corresponding author
}

\section{To cite this article:}

Ana Antonia Collado, Sandra Fachelli, Zi Yu Liu. Access to the labor market of the graduates of the Faculty of Law of the University of Barcelona (Spain). Science Innovation. Vol. 4, No. 6, 2016, pp. 303-310. doi: 10.11648/j.si.20160406.20

Received: November 15, 2016; Accepted: December 27, 2016; Published: December 30, 2016

\begin{abstract}
The objective of this article is to analyse the degree and characteristics of the labour insertion of the graduates of the Faculty of Law when comparing them with the rest of graduates of the University of Barcelona, taking into account the sex, labour situation during the university pathway and the average academic grade. The data comes from the Labour Insertion Survey to the graduated population of Catalan universities carried out by the Agency for Quality of the University System of Catalonia (AQU) in 2014. The sample used corresponds to 3,425 university graduates in the University of Barcelona. The technique used is bivariate and multivariate analysis. For the comparative analysis we used contingency tables and for the explanatory, bivariate logistic regression. The results show that the employment situation of all graduates of the Faculty of Law is characterized by a high degree of occupation. Stability in employment on fixed contracts is higher in men than in women; the full-time work is predominantly homogeneous in both groups, and the monthly salary shows an inequality between women and men, the women have the most precarious conditions. In this way, the results obtained allow us to validate two hypotheses and refute a third one. In the first instance, there is a positive relationship between the degree of occupation and the academic grade; secondly, gender inequality is confirmed in favour of men in terms of working conditions. Finally, unlike expected, it is observed that there is a certain adequacy or matching between the work done and the training received at the university.
\end{abstract}

Keywords: Labour Insertion, Graduates Employment, Gender Inequality, Labour Conditions, Labour Market, Faculty of Law, University of Barcelona

\section{巴塞罗那大学法律系毕业生的就业情况}

Ana Antonia Collado ${ }^{1 *}$, Sandra Fachelli ${ }^{2}$, 刘子瑜 ${ }^{3}$

1社会学部, 巴塞罗那大学, 巴塞罗那, 西班牙

${ }^{2}$ 社会学部, 巴塞罗那自治大学, 贝拉特拉, 西班牙

${ }^{3}$ 法律系, 巴塞罗那大学, 巴塞罗那, 西班牙

邮箱

antoniacollado@ub. edu (A. A. Collado), Sandra.Fache1li@uab.cat (S. Fache1li), 1iuziyu20@hotmail.com(刘子瑜)

摘要：这篇文章的目的是分析在性别、大学期间工作情况以及平均分的影响下，巴塞罗那大学法律系的毕业生就业情 况的程度和特点以及与该大学其他院系的毕业生就业情况的比较。所有资源都来源于由加泰罗尼亚地区大学质量体系 研究局制定的2014年加泰罗尼亚所有大学毕业人口的就业调差问卷。样本来源于巴塞罗那大学3425名毕业生。我们所 
采用的分析技术是多元化和复合式的。比较分析方面我们使用列联表来得出结论，而解释分析方面则采用二元逻辑回 归的方式来体现。从结果中我们不难看出法律系的毕业生的整体就业状况成高就业率的状态。在工作的稳定方面, 男 性毕业生拥有固定合同的数量高于拥有固定合同的女性毕业生; 全职工作的工作方式在这两组人群中占主导方式，在 月收入方面在男毕业生和女毕业生出现一个不平等现象，女性有更多不利因素。所以，以上所得出的结果可以验证两 个假设以及反驳一个假设。首先发现在就业占有率和在校成绩二者之间成正比关系，其次，证实在工作条件方面有利 于男性的性别不平等性。最后, 不同于预期, 我们观察到在我们所从事过的工作与大学期间接受的教育之间有一些适 合性。

关键词：就业，毕业生就业，性别不平等，工作条件，劳动力市场，法律系，巴塞罗那大学

\section{1. 引言}

从影响用工需求的社会经济环境状况以及有可能进 入劳动力市场的大学毕业生方面来看, 就业是一个需要考 虑到多种因素的复杂过程.

因此, 有必要去研究大学在青年就业方面所扮演的角 色。大学教育不仅仅是提高国民教育水平、传播正确的价 值观, 同时也会改善毕业生的就业前景[1]。在这种情况下， 想知道。大学毕业生在就业方面有什么影响? 本科毕业生 的工作条件怎么样?

基于大学与就业市场之间联系的重要性, 这项研究分 析了巴塞罗那大学法律系2009-2010届毕业生与该校同届 的其他院系毕业生的就业状况, 以及两者的比较。调查的 数据来源于由加泰罗尼亚地区大学质量体系研究局制定 的2014年加泰罗尼亚所有大学毕业人口的就业调差问卷。

在这个研究中, 我们提出一个源自于多个具体目标的 总体目标。这个总体目标是对法律系毕业生与该校其他院 系毕业生的就业程度和特点的比较分析。

具体目标为以下内容:

1 法律系毕业生已就业学生的工作条件与该校其他 系毕业生作比较, 同时考虑工作合同的种类、工 作时间、薪资、职业级别、性别和所从事的工作 与受教育水平之间的合适性等等相关因素对工作 条件的影响。

2 在性别划分为前提下, 以比较分析的方式研究法 律系毕业生以及其他院系毕业生的社会出身 (父 母的受教育水平)。

以下是基于这个研究而引出的假设。

1 法律系毕业生的工作条件与该大学其他院系的毕 业生相似。

2 大学学习使社会家庭出身带来的影响最小化, 尤 其是在女性毕业生当中效果看起来更加明显。

\section{2. 理论框架}

从上世纪五十年代开始, 全民的教育水平已经开始产 生持续的增长。尤其是最近几十年, 越来越多的年轻人逐 渐进入大学学习, 目前女性群体比重的增长达到了一个新 高度。整个西班牙在过去十年中多于三分之一的青年人已 经开始了大学生活。这既是量变又是质变。从18到 22 岁的 大学教育的净增长率由 1992 学年的19\%到 $23 \%$ 之间增长到
2010 年的 $28 \%$ 到 $32 \%$ 之间 [2],[3]。另一方面, 这一变化同 样可以在学生类型的质量方面被发现: 更加的女性化, 不 同的目的性以及不同的年龄段 [4]。这些特征以及其他相似 特征作为“大学民主化”这一熟知的现象被突出显示出来, 并且提出了一个涉及到目前和将来大学教育战略来适应 这些特点变化[5]。

这些变化是规划我们分析每一个专业的现状特别是 提醒如果变化被认为是结构上的或者紧跟教育扩展这一 被熟知的过程开始的潮流趋势。

Fachelli\&Planas[6]向我们展示了在持两种完全不同 的观点的作者之中产生的争论。一方面, 有些作者认为青 年人的“过量教育”是比较危险的, 另一方面, 一部分支持 提高教育水平。面对这些相对立的观点，应该发问毕业生 在通过大学文凭资格进入劳动力市场时大学机构起到什 么样的作用; 因此再一次回到这个重要的问题, 相较于没 有进入大学学习的青年, 大学教育是否在就业方面有所帮 助?

多样的研究显示出如何提高教育水平, 尤其是达到一 个本科学位，是进入劳动力市场的最有效的方法之一，同 样的也是上升到更高的社会阶层的有效手段之一[7],[8]。

对于大多数人来说, 大学这一步骤代表了向成年人生 活的过渡, 从而向劳动世界的过渡。因此, 这是一个借助 多种方式 (例如就业指导办公室, 企业论坛以及其他积极 主动的方式) 指引毕业生职业生涯的复杂过程, 但是同样 也是社会和经济的环境等多重因素共同作用影响的结果, 根据作者Cantonnet, Berbegal\&Ochoa[9]在他的著作里对 毕业生的需求也进行了条件限定。

总的来说, 就业这一概念被习惯用于展示人类进入经 济活动的这一过程。从José Navarro[8]的观点来看, 我们] 提及的就业是由许多个人因素的综合来确定的, 例如主要 的有教育和职业培训, 寻找工作的策略, 寻找工作所花费 的时间, 职业期望以及薪资期望。如此, 得到一个大学文 凭是提高进入劳动力市场可能性的方法之一。

展现西班牙劳动力市场的一个重要特点是具有把临 时合同的使用作为工作岗位收入机制的趋势的明显特征 以及具有就业延误与上一代人的工作转换相比较的特征 [8]。

另外一个具有极大意义的主题是社会出身在就业方 面的影响。对于这个领域的研究文献是十分丰富的。一方 面着重指出在社会优势上, 父辈的资本会转移到子女身上, 并且同样可以在不同方式的教育系统中可见[10],[11],[12], 发现阶层越高的子女选择更高的学术方向发展的趋势同 
时阶层越低的子女会在职业类型的方面有更多的规划, 例 如技术领域相关的工作 [13]。同样可以被了解到的是选择 学习什么样的专业与这个学生以及其家庭成员因学业有 成而得到的机会和经历有关系。周期长的专业意味这一个 多年的长期投资, 这对于那些之前都没有良好的大学前期 学术背景的人有可能存在失败的风险[14],[15]。

同样的存在众多的文献来研究本科学历在社会组成 方面所产生的作用[16]。从假设角度出发, 子女有可能更 多的在与父母职业相似的领域选择自己的专业, 比如Van de Werfhorst\&Luijkx[13], 曾经调查了在荷兰社会中父母 的职业对于其子女在专业选择方面的影响。这些研究者确 实发现了在社会阶层和受教育水平关系的作用下, 子女通 常会选择与父辈职业关系密切的专业来学习。

最终, 似乎证明了在大学内部依然运行按照社会组成 情况的学历分层机制[16],[12], 但是个人因素也同样会在 其中产生影响。Troiano\&Elias[15]提出一个在专业选择方 面会包含发挥作用的因素的模式, 这些因素既源于个人同 时又于集体。专业选择是在偏好（个人、社会和意愿期望） 与对这个选择的相对成本评估所产生的风险（直接成本, 间接成本和机会成本, 同样也有收入) 以及成功概率三者 平衡的结果 (可能的选择, 个人能力, 期限和预期困难)。

那么, 当开始分析就业结果的时, 一通过大学的篮选 便逐渐获得了大学学历, 社会出身的影响力就看起来变得 微弱了。Carabaña在他的很多研究分析中就是持这种观点 [17],[18], 同时其确定性与可靠性也被展示出来, 当一个 人成功的进入大学, 如果他能毕业, 那么就会使他的社会 出身所带来的影响比重最小化。也就是说通过大学的学习 有可能软化阶级冲突 [19]。同样的, 在加泰罗尼亚地区所 有毕业生在之后的工作方面我们可以得到一个结论, 大多 数毕业生的就业情况仍然处于学历的影响之下, 并没有发 现社会出身在其中起到十分重要的作用[20]。

最后, 经济危机的环境也促使我们发问, 面对着我们 正在经历的长期经济危机, 毕业生们应该进行什么样的活 动来更好的应对: 学习? 或者说目前我们都还没有停留在 这个方面, 最新的数据允许证明这个猜想。事实是在 2014 年加泰罗尼亚地区公立大学的 $76,8 \%$ 的毕业生继续他们的 学习, 其中 $40 \%$ 选择硕士教育[21]。另外一个我们可以考 虑的因素是所从事的工作质量越高职业级别越高的毕业 生展现的对大学教育与工作应用的之间关系的评价水平 越高[22]。

\section{3. 方法和数据分析类型}

使用的方法是定量分析法, 通过列联表和线性回归的 统计技术，这个方法使估测毕业生就业现象成为可能。列 联表可以被认为是作为分析定性变量的具有重要性特点 的手段以及为社会分析员在各种现象当中关系的可以快 速阅读提供技巧帮助。用这个方法可以确定在变量里是否 存在关联以及这是一个怎样的联系。或者相反, 并不存在 这个关联性, 而且我们可以说在这些变量之间都是相互独 立的。如果存在关联性, 伴随着介于 0 至 1 之间变化的
Cramer V系数, 越渐近 1 表示行列关联很强, 接近 0 表示关 联很弱或不相关, 这个联系力将能够被估测 ${ }^{1}[23]$ 。因此, 根据毕业的地点对列联表进行过分析来观察在研究中参 与的定性变量之间的关联水平。

在线性回归的分析中两个假设都将会被测验, 为此我 们将使用两组 (法律系和巴塞罗那大学其他系) 的学生的 年收入作为因变量 $\left(Y_{i}\right)$ 。自变量是性别（男、女）, 工作 合同类型 (固定合同, 临时合同, 自主合同等其他合同), 工作时间（全职, 兼职), 工作职能（大学专业方面, 非 大学专业方面), 职业级别（高级, 中级或者低级) 以及 父母的受教育水平（初级, 中级或者高级）。

$$
Y_{i}=\alpha+\beta_{1} X_{\text {女性 }}+\beta_{2} X_{\text {固定 }}+\beta_{3} X_{\text {兼职 }}+\beta_{4} X_{\text {非大学的 }}+\beta_{5} X_{\text {低 }}+\beta_{6} X_{\text {小学 }}+\varepsilon_{i}
$$

最后, 关于所使用的变量“职业级别”我们来做一份方法 说明。

我们根据由 Fachelli\&Montolio[22]制定的分类，把来源 于国家职业分类应用的九大职业分成 3 组, 如表 1 所示。

表1 来源于国家职业分类应用的职业级别变量分组。

\begin{tabular}{ll}
\hline 职业等级 & 国家职业分类(CNO) \\
\hline 高 & 1负责人和经理 \\
& 2专家,科学家和知识分子 \\
中 & 3技术人员;基础专业人员 \\
& 4会计人员,办公室行政和其他人员 \\
& 5服务人员,餐馆饮食人员,安保和售货员 \\
& 6农业方面的熟练工人,牧场人员,林业人员和渔业人员 \\
& 7工匠和制造业熟练工人和建筑工人 \\
低 & 8机器和设备的操作员和装配工 \\
& 9基础工作 \\
\hline
\end{tabular}

进行这项调查研究所使用的数据来源于由加泰罗尼 亚地区大学质量体系研究局 (以下称为 $\mathrm{AQU}^{2}$ ) 制定的 2014 年加泰罗尼亚所有大学毕业人口的就业调差问卷

从2001年开始, AQU在加泰罗尼亚社会理事会的帮助 下对大学毕业生毕业三年之后的就业状况进行三年一次 的调查活动, 以此来了解就业的时间、质量和就业方式以 及对工作的满意程度。

这个 2014 年出版的第五个版本的研究总体是 2009-2010学年所有公立和私立大学和 24 个附属中心（比 前一版多出两个附属中心) 的毕业生的总和, 即 31279 人 次。样本总数是 17337 位毕业生, 占总体人数的 $55 \%$, 抽 样误差范围为 $0.51 \%{ }^{3}$ [24].

然而这个统计研究的范围是由巴塞罗那大学共计 7759位毕业生组成的, 研究该校法律系的917名毕业生与 其他院系学生的不同。二者的样本分别是 3076 人和 349 人 （图表2）。

1在社会现象当中Cramer V系数代表的关联力达到了 0.6

2加泰罗尼亚地区大学质量体系研究局, 是推动和促进加泰罗尼亚大学 教育质量的主要机构具有对整个加泰罗尼亚地区在大学和高级教育中 心范围内针对质量的评估、确认和委托的目标.

32014年第五版的调查问卷的总体、样本、回答比率和样本误差的数据 均被展示于A附属部分. 
表2 总体, 样本, 回答的百分比法律系的样本误差范围。

\begin{tabular}{lllll}
\hline 学位 & 总体 & 样本 & 回答的百分比 & 样本误差 \\
\hline 公共行政管理 & 58 & 37 & $63,79 \%$ & $9,78 \%$ \\
工作关系 & 256 & 97 & $37,89 \%$ & $7,86 \%$ \\
犯罪学 & 122 & 68 & $55,74 \%$ & $7,94 \%$ \\
法律 & 435 & 114 & $26,21 \%$ & $7,89 \%$ \\
政治科学 & 46 & 33 & $71,74 \%$ & $9,17 \%$ \\
总和 & 917 & 349 & $38,06 \%$ & $8,87 \%$ \\
\hline
\end{tabular}

\section{4. 结果}

\section{1. 就业, 失业和不工作}

在这份报告里我们着重研究了大学毕业生与就业市 场的之间所存在的关系类型。通过对正在进行有偿工作 的、目前失业正在寻找工作的以及处在职场范围之外的 毕业生们的逐渐了解，一个整体性观点均衡的概括了毕 业生目前的就业程度和状况。
巴塞罗那的毕业生在毕业三年之后的就业程度被认 为是非常高的, 因为 $83 \%$ 的学生是处于工作的状态。关于 失业率, 是成降低趋势的, 因为只有 $12 \%$ 的毕业生失业, 每 20 个人当中只有一个是不工作的, 换句话说，就是处于 就业市场之外的（既不工作也不寻找工作）。在法律系和 其他院系毕业生中，这些数据都是相似的。正如图表3所 体现的那样, 尽管这样, 在两组数据里并不存在一个重要 的差异。

表 3 巴塞罗那大学所有毕业生的就业状况。

\begin{tabular}{|c|c|c|c|c|c|c|c|c|}
\hline \multirow{2}{*}{ 工作状况 } & \multicolumn{8}{|l|}{ 2014年 } \\
\hline & 法律系 & & 其他院系 & & 总和 & & Chi-Square & Cramer V系数 \\
\hline 目刖上作情况 & & & & & & & $2,315 \mathrm{a}(0,314)$ & ------ \\
\hline 就业 & 299 & $85,70 \%$ & 2.545 & $82,70 \%$ & 2. 844 & $83,00 \%$ & & \\
\hline 失业 & 34 & $9,70 \%$ & 385 & $12,50 \%$ & 419 & $12,20 \%$ & & \\
\hline 不工作 & 16 & $4,60 \%$ & 146 & $4,75 \%$ & 162 & $4,70 \%$ & & \\
\hline 失业状况 & & & & & & & $2,247 \mathrm{a}(0,134)$ & ------ \\
\hline 未失业 & 315 & $90,30 \%$ & 2.691 & $87,50 \%$ & 3. 006 & $87,80 \%$ & & \\
\hline 失业 & 34 & $9,70 \%$ & 385 & $12,50 \%$ & 419 & $12,20 \%$ & & \\
\hline
\end{tabular}

\section{2. 巴塞罗那大学法律系毕业生和其他院系毕业生的工} 作条件的区别

在这一章里通过以下变量可以了解到正在工作的毕 业生其工作条件的质量水平：工作合同类型，工作时间类 型，职业级别，月收入以及所从事的工作与受教育水平的
合适性（图表4）。关于工作合同类型, 在巴塞罗那大学 所有正在工作的毕业生之中, 有多于一半的人表明拥有固 定合同，同时有三分之一的人具有临时合同。自主就业占 毕业生的 $10 \%$ ，实习生的比例很少（5\%），无合同就业的 比例更加微不足道。

表4 巴塞罗那大学法律系毕业生和其他院系毕业生的工作条件的区别。

\begin{tabular}{|c|c|c|c|c|c|c|c|c|}
\hline \multirow{2}{*}{ 工作状况 } & \multicolumn{8}{|l|}{ 2014年 } \\
\hline & 法律系 & & 其他院系 & & 总和 & & Chi-Square & Cramer V系数 \\
\hline 女性 & 163 & $54,5 \%$ & 1. 178 & $70,3 \%$ & 1. 341 & $60,0 \%$ & & \\
\hline 男性 & 136 & $45,5 \%$ & 757 & $29,7 \%$ & 893 & $40,0 \%$ & & \\
\hline 工作合同类型 & & & & & & & $66,720 \mathrm{c}(0,000)$ & $0,153(0,000)$ \\
\hline 固定 & 199 & $66,8 \%$ & 1. 279 & $50,3 \%$ & 1. 478 & $52,0 \%$ & & \\
\hline 临时 & 43 & $14,4 \%$ & 865 & $34,0 \%$ & 908 & $32,0 \%$ & & \\
\hline 自主就业 & 49 & $16,4 \%$ & 237 & $9,3 \%$ & 286 & $10,1 \%$ & & \\
\hline 实习生 & 6 & $2,0 \%$ & 128 & $5,0 \%$ & 134 & $4,7 \%$ & & \\
\hline 无合同 & 1 & $0,3 \%$ & 34 & $1,3 \%$ & 35 & $1,2 \%$ & & \\
\hline 工作时间类型 & & & & & & & $38,778 \mathrm{~d}(0,000)$ & $0,120(0,000)$ \\
\hline 全职工作 & 252 & $86,0 \%$ & 1. 652 & $68,4 \%$ & 1.904 & $70,31 \%$ & & \\
\hline 兼职工作 & 41 & $14,0 \%$ & 763 & $31,6 \%$ & 804 & $29,7 \%$ & & \\
\hline 职业等级 & & & & & & & $26,578 \mathrm{a}(0,000)$ & $0,090(0,000)$ \\
\hline
\end{tabular}




\begin{tabular}{|c|c|c|c|c|c|c|c|c|}
\hline \multirow{2}{*}{ 工作状况 } & \multicolumn{8}{|c|}{ 2014年 } \\
\hline & \multicolumn{2}{|c|}{ 法律系 } & \multicolumn{2}{|c|}{ 其他院系 } & \multicolumn{2}{|l|}{ 总和 } & \multirow[t]{2}{*}{ Chi-Square } & \multirow[t]{2}{*}{ Cramer V系数 } \\
\hline 高 & 172 & $51,2 \%$ & 1.913 & $65,1 \%$ & 2.085 & $63,6 \%$ & & \\
\hline 中 & 112 & $33,3 \%$ & 743 & $25,3 \%$ & 855 & $26,1 \%$ & & \\
\hline 低 & 52 & $15,5 \%$ & 284 & $9,7 \%$ & 336 & $10,3 \%$ & & \\
\hline 月收入 & & & & & & & $32.259 \mathrm{c}(0,000)$ & $0,110(0,000)$ \\
\hline 一千 & 82 & $30,0 \%$ & 1. 116 & $46,3 \%$ & 1. 198 & $44,6 \%$ & & \\
\hline 二千 & 103 & $37,7 \%$ & 807 & $33,5 \%$ & 910 & $33,9 \%$ & & \\
\hline 高于二千 & 88 & $32,2 \%$ & 488 & $20,2 \%$ & 576 & $21,5 \%$ & & \\
\hline 所需要的 & & & & & & & $21,911 \mathrm{~d}(0,000)$ & $0,088(0,000)$ \\
\hline 非大学功能 & 105 & $35,1 \%$ & 582 & $22,9 \%$ & 687 & $24,2 \%$ & & \\
\hline 大学功能 & 194 & $64,9 \%$ & 1.963 & $77,1 \%$ & 2. 157 & $75,8 \%$ & & \\
\hline
\end{tabular}

比较法律系与其他院系的毕业生, 法律系的拥有固定 合同和自主就业合同的毕业生数量占优势。在第一种情况 下，法律系有三分之二的毕业生拥有固定合同，而其他院 系拥有相同类型合同的人只占 $50 \%$ 。在第二种情况下， $16 \%$ 的法律系毕业生自主就业, 而其他院系自主就业的比例只 有 $9 \%$ 。巴塞罗那其他院系的毕业生的工作稳定性相对较差: $34 \%$ 的人有临时合同， $5 \%$ 的实习生和 $1.3 \%$ 的无合同工作者。

其次, 通过区分全职工作的和兼职工作的毕业生来分 析工作时间这个变量。正如我们在图表4所看到的, 在巴 塞罗那大学所有毕业生当中有 $70 \%$ 的人是全职工作, 而从 事兼职的只占总人数的三分之一。

关于工作时间的类型，通过观察到的法律系毕业生全 职工作的人数比例是最高可以看出法律系与其他院系的 毕业生相比所存在的差异是非常重要的。法律系毕业的人 的 $86 \%$ 正在从事全职工作, 而其他院系的学生只占 $68 \%$ 。相 反的, 从事兼职工作的情况在其他院系的毕业生当中更加 普遍：兼职比例为三分之一。

紧接着, 通过毕业生职业等级的评估来对就业质量 的数据分析, 在巴塞罗那其他院系毕业生中展示出一个 清晰的优势。这个差异尤其体现在越高的等级当中, 但 是当然，也体现在越低的等级之中。首先，法律系 $50 \%$ 的毕业生从事高级别工作，而其他院系有三分之二的人 处于相同情况。其次, 低成绩的在法律系毕业生当中的 出现率是 15\%, 而在其他院系里只有 $9 \%$ 。

月收入同样也是衡量分析工作条件质量的指标。可以指 出的是在所有已毕业的工作者中, 月收入在 1 千欧元左右的 将近占总人数的一半 $(45 \%)$, 只有三分之一的人可以月赚2 千欧元左右，最后，那些月工资更高的人的比例为 $22 \%$ 。

在比较法律系和其他院系毕业生的收入情况时, 可 以发现在两组中存在着不一样的分配情况, 法律系学生
的收入形势是更有利的。其他院系毕业生大概有一半的 人（46\%）月收入最低（每个月大概在一千欧元左右）, 而法律系的比例较其低了16个百分点（30\%）。相反的, 法律系有大概 $70 \%$ 的毕业生的月收入达到了平均值 (2000 欧元）或高于平均值（多于 2000 欧元）, 而其他院系的人 只有 $54 \%$ 达到相同水平。

一个应该考虑到的可以用来解释就业质量的重要因 素是所从事的工作是否与其受教育水平相符合。换句话 说就是拥有本科学历这个事实。这可以说是一个积极的 关系, 因为 $76 \%$ 的巴萨罗那大学毕业生从事的工作职能与 其大学文凭是相一致的。但是与其他院系的毕业生相比, 法律系的学生处于一个更加不利的情况中。65\%的法律系 学生正在从事的工作与他们的专业有关系, 而其他院系 的人数比例则达到了 $76 \%$ 。

\section{3. 父辈的教育水平 (法律系和该校其他院系)}

个体的社会出身是对能够进入大学学习产生影响的 一个因素。也就是说, 社会阶层, 职业等级, 或者父辈的 受教育水平可能在子女的大学学习程度以及学历类型方 面产生作用。

在这个研究中, 我们根据父辈的受教育水平来展示毕 业生的社会出身（图表5）。所得到的结果表明巴塞罗那大 学 $67 \%$ 的毕业生的父母拥有中级或者高级的教育水平, 而剩 下的三分之一的学生, 他们的父母的教育水平为初级。

通过观察毕业于法律系和其他院系的学生的数据可 以发现在两者之间并不存在一个重要的差异

针对于法律系毕业生同样也分析了父辈受教育水平 与毕业生本身性别 (男性和女性) 之间的关系。但这个关 系并不十分重要, 两者均为独立存在的。

表5 毕业生父母的教育水平的影响作用。

\begin{tabular}{lllllllll}
\hline \multirow{2}{*}{ 父母教育 } & \multicolumn{1}{l}{2014 年 } & \multicolumn{1}{l}{ C } & & & & \\
\cline { 2 - 8 } & 法律系 & & 其他院系 & & 总和 & & Chi-Square & Cramer V系数 \\
\hline 初级 & 128 & $37,0 \%$ & 1.009 & $33,0 \%$ & 1.137 & $33,4 \%$ & $4,961 a(0,084)$ & ---- \\
中级 & 106 & $30,6 \%$ & 876 & $28,6 \%$ & 982 & $28,8 \%$ & & \\
高级 & 112 & $32,4 \%$ & 1.176 & $38,4 \%$ & 1.288 & $37,8 \%$ & & \\
\hline
\end{tabular}




\section{4. 从属关系的研究}

接下来展示从属关系的分析结果。（图表6）因变量 是工作者的年收入。采用线性回归的方法来比较两组的结 果: 巴塞罗那大学法律系毕业生和该校其他院系的毕业生 （法律系的毕业生占该校总体毕业生的 $10.2 \%$ ）。

第一个我们应该说明的是巴塞罗那法律系的毕业生 与该校其他院系的毕业生之间存在差异。在法律系毕业生 收入方面产生影响的特点是以下几条:

a 女性收入少于男性收入。

b 一固定合同工作的收入要高于以临时合同工作的人, 自主就业的收入要更少于前两者。

c 全职工作的收入高于兼职。

$\mathrm{d}$ 从事大学职能的工作的人的收入要高于从事非大学 职能的人。 e 根据父母的受教育水平并没有发现子女在职业等级 方面重要的不同。

$\mathrm{f}$ 在巴塞罗那大学其他院系毕业生的收入中产生作用 的特点, 我们可以按照以下方式进行综合概括:

$\mathrm{g}$ 女性比男性的收入少。

$\mathrm{h}$ 拥有固定合同的人的收入多于拥有临时合同的人, 但 是拥有其他种合同的人与有临时合同的人收入没有 区别。

i 从事全职工作的人的收入高于从事兼职工作的人。

$j$ 进行具有大学职能工作的人的收入高于从事不具有 大学职能工作的人。

$\mathrm{k}$ 在职业等级方面, 从事中级或者高级的人的收入高于 从事低级别的人。

1 父母具有中级教育水平的子女收入低于父母教育水 平为低级的子女收入, 最后, 针对父母学历高低的比 较, 在毕业生的收入方面没有发现重要的差异。

表6 毕业生的就业 (法律系和其他院系) 根据年收入状况。

\begin{tabular}{|c|c|c|c|c|c|}
\hline \multirow{3}{*}{ 自变量 } & \multicolumn{5}{|c|}{ 法律系的毕业生 } \\
\hline & \multicolumn{2}{|c|}{ 非标准化系数 } & \multirow{2}{*}{$\begin{array}{l}\text { 典型化系数. } \\
\text { Beta }\end{array}$} & \multirow{2}{*}{$\mathrm{t}$} & \multirow{2}{*}{ 有意义的 } \\
\hline & B & 标准误差 & & & \\
\hline (Constant) & 13668,9 & 1882,2 & & 7,3 & 0,00 \\
\hline \multicolumn{6}{|l|}{ 性别: 男性 (*) } \\
\hline 女性 & $-5526,1$ & 941,8 & $-0,3$ & $-5,9$ & 0,00 \\
\hline \multicolumn{6}{|l|}{ 合同:临时的 $(*)$} \\
\hline 固定 & 3588,1 & 1183,0 & 0,2 & 3,0 & 0,00 \\
\hline 自主就业 & $-3507,7$ & 1644,5 & $-0,1$ & $-2,1$ & 0,03 \\
\hline $\begin{array}{l}\text { 其他或者无合同 } \\
\text { 工作时间: 兼职 (*) }\end{array}$ & $-1680,1$ & 4715,0 & 0,0 & $-0,4$ & 0,72 \\
\hline 全职 & 8711,7 & 1321,5 & 0,3 & 6,6 & 0,00 \\
\hline \multicolumn{6}{|l|}{ 进行的功能: 非大学的 (*) } \\
\hline 大学的 & 3622,0 & 1130,8 & 0,2 & 3,2 & 0,00 \\
\hline \multicolumn{6}{|l|}{ 职业等级: 低 $(*)$} \\
\hline 中 & $-2759,7$ & 1435,3 & $-0,1$ & $-1,9$ & 0,06 \\
\hline 高 & $-1632,5$ & 1512,1 & $-0,1$ & $-1,1$ & 0,28 \\
\hline \multicolumn{6}{|l|}{ 父母的教育水平: 初级 $(*)$} \\
\hline 中级 & 523,2 & 1105,7 & 0,0 & 0,5 & 0,64 \\
\hline 高级 & 197,4 & 1135,6 & 0,0 & 0,2 & 0,86 \\
\hline \multicolumn{6}{|l|}{$\mathrm{R}^{2}$ 修改的: 0,327} \\
\hline n=301毕业生 & & & & & \\
\hline 因变量: 年收入 (范围的中点) & & & & & \\
\hline
\end{tabular}

表6 继续。

\begin{tabular}{|c|c|c|c|c|c|}
\hline \multirow{3}{*}{ 自变量 } & \multicolumn{5}{|c|}{ 其他院系的毕业生 } \\
\hline & \multirow{2}{*}{$\begin{array}{l}\text { 非标准化系数 } \\
\mathrm{B}\end{array}$} & \multicolumn{2}{|c|}{ 典型化系数. } & \multirow{2}{*}{$\mathrm{t}$} & \multirow{2}{*}{ 有意义的 } \\
\hline & & 标准误差 & Beta & & \\
\hline $\begin{array}{l}\text { (Constant) } \\
\text { 性别: 男性 (*) }\end{array}$ & 8040,4 & 551,8 & & 14,6 & 0,00 \\
\hline $\begin{array}{l}\text { 女性 } \\
\text { 合同:临时的 }(*)\end{array}$ & $-2489,9$ & 292,5 & $-0,1$ & $-8,5$ & 0,00 \\
\hline 固定 & 3768,6 & 288,0 & 0,2 & 13,1 & 0,00 \\
\hline 自主就业 & 719,2 & 503,1 & 0,0 & 1,4 & 0,15 \\
\hline $\begin{array}{l}\text { 其他或者无合同 } \\
\text { 工作时间: 兼职 }(*)\end{array}$ & $-1700,4$ & 975,4 & 0,0 & $-1,7$ & 0,08 \\
\hline $\begin{array}{l}\text { 全职 } \\
\text { 进行的功能: 非大学的 (*) }\end{array}$ & 7006,5 & 295,9 & 0,4 & 23,7 & 0,00 \\
\hline
\end{tabular}




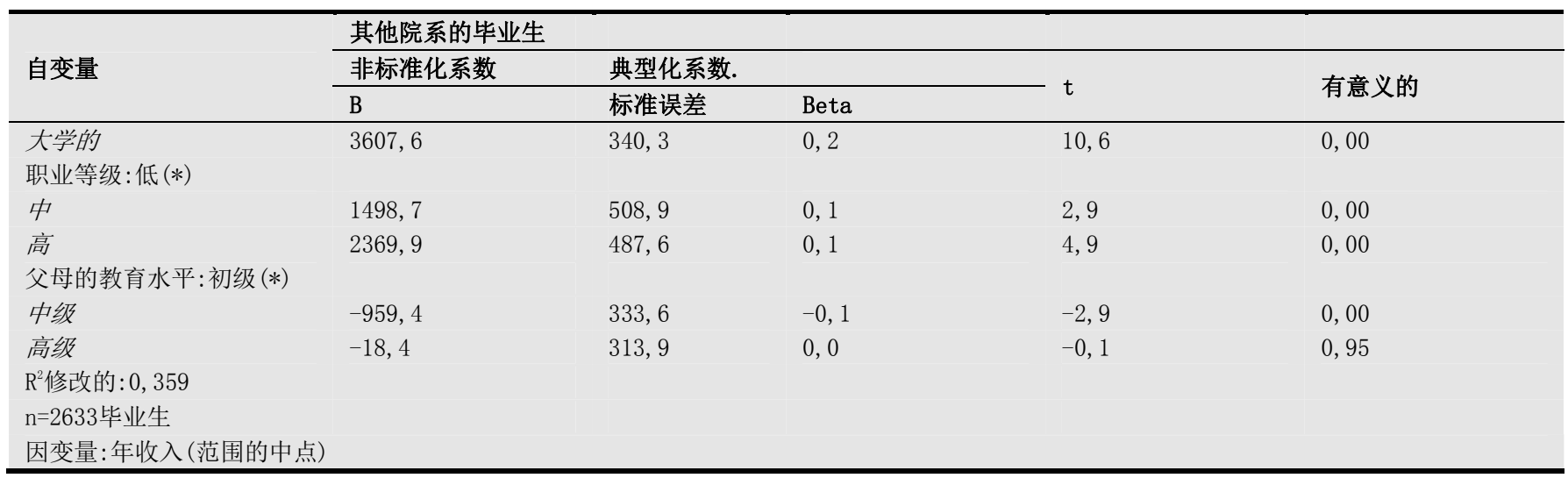

\section{5. 结论}

这篇文章展示了通过区分巴塞罗那大学法律系与其 他院系毕业生在劳动力市场的就业程度, 工作条件、家庭 的社会出身以及父辈的受教育水平等方面进行的一个比 较分析。

一些重要的发现表明了在两组中高就业率的相似性: 五分之四左右的毕业生拥有工作。

然而在法律系毕业生和其他院系毕业生之中针对工 作条件质量方面依然出现了重要的差异。在以下方面法律 系毕业生的工作条件具有更大的优势: 具有更大的工作稳 定性, 全职工作率和收入都是更高的。然而, 其他院系毕 业生在职业等级方面更占优势, 并且工作职能与大学教育 水平的协调性也更高。

对于父辈的教育水平, 在两组（法律系和其他院系） 中的关系并不重要, 同时也不认为在法律系学生中与性别 变量的关系是具有重大意义的。

正如我们在理论框架中所看到的那样，在一般情况 下, 青年人教育水平的提高, 尤其是大学教育, 尽管不 能解决受经济危机影响的所有问题, 但可以提高年轻人 口尤其是大学毕业生的就业程度以及改善其工作条件。

针对巴塞罗那大学毕业生的具体情况, 通过从属关系 的分析, 在就业方面展现出众多相似性, 男性工作者、具 有固定合同的、从事全职工作的以及其工作是进行大学职 能的工作者都会有高额收入。最后, 对于社会出身, 正如 我们在理论章节看到的那样, 并不存在父母学历越高则子 女收入水平越高的关系, 因此, 当研究就业市场的收入状 况时, 社会出身的影响没有再一次在巴塞罗那大学毕业生 中被找到。

\section{致谢}

该研究项目是在由Antonia Collado指导的 “法律系 毕业生就业状况以及国际和巴塞罗那大学经济和企业管 理系的经验的转移分析” (REDICE16-1682) 这个调查项目 背景下进行的。同样在接收由 2020 年研究和创新计划资助 的欧洲项目Red INCASI的背景下展开的, 由Marie Sk子 odowska-Curie GA指导, Pedro López-Roldán协助。“该 文章仅反映作者观点, 对文章内容的使用机构不作任何保 证或承诺”。

\section{参考文献}

[1] G. García, "La inserció laborals dels graduats universitaris: la qüestió de la sobrequalificació i el desajust formatiu, " Nota d'economia, 2005, vol. 81, pp. 99-119.

[2] Instituto Nacional de Calidad y Evaluación, Sistema estatal de indicadores de la educación: 2000, 2000, Madrid: MECD.

[3] Ministerio de Educación, Cultura y Deporte, Las cifras de la educación en España. Curso 2010-11, 2013, Madrid: MECD.

[4] A. Ariño \& R. Llopiz, ¿Universidad sin clases? Condiciones de vida de los estudiantes universitarios en España (Eurostudent IV), 2011, Madrid: Ministerio de Educación. Secretaría General de Universidades.

[5] A. Corominas \& V. Sacristán, “Las encrucijadas estratégicas de la universidad pública española, ” 2011, Revista de Educación, 355, pp. 57-81.

[6] S. Fachelli \& J. Planas, “Inserción profesional y movilidad intergeneracional de los universitarios: de la expansión a la crisis, ” 2014, Revista Española de Sociología (RES), vol 21, pp. 69-98. http://ddd. uab. cat/pub/artpub/2014/125654/revespso c_a2014n21p69iSPA.pdf.

[7] S. Fachelli \& J. Planas, “Equidad y movilidad intergeneracional de los titulados universitarios catalanes, ”2011, PAPERS, Revista de Sociologia. Vol 96/4, pp. 1307-1331.

[8] J. Navarro-Cende jas, Universidad y mercado de trabajo en Cataluña: un análisis de la inserción laboral de los titulados universitarios, 2013, Tesis doctoral. Universidad Autónoma de Barcelona. http://ddd. uab. cat/pub/tesis/2014/hdl_10803_134648 / jnc1de1. pdf. 
[9] M. L. Cantonnet, J. Berbegal \& C. Ochoa "Formación académica e inserción laboral: ¿Una asignatura pendiente?, " Jornadas de la Asociación de Economía de la Educación. Investigaciones de la Economía de la Educación (La Coruna: 2013) [en línea]. Barcelona: Universitat Politècnica de Catalunya, Portal del coneixement obert de la UPC. http://upcommons. upc. edu/handle/2117/21522.

[10] J. Calmand \& D. Epiphane, 2012, “L' insertion professionnelle après des études supérieures: des diplômés plus égaux que d' autres, ” Formation Emploi, 117, pp. $11-28$.

[11] S. Lucas, "Effectively maintained inequality: Education transitions, track mobility, and social background effects, ” 2001, American Journal of Sociology, 106 (6), pp. $1642-1690$.

[12] M. Triventi, “Stratification in Higher Education and its relationship with social inequality. A comparative study of 11 European Countries, ” 2011, European Sociological review, pp. 1-14.

[13] H. Van de Werfhorst \& R. Luijkx, “Educational Field of Study and Social Mobility: Disaggregating Social Origin and Education, ” 2010, Sociology, 44 (4), pp. $695-715$.

[14] M. Salas, "La demanda de Educación Superior: un análisis microeconómico con datos de corte transversal, ” 2005, Revista de Educación, 337, 327 349.

[15] H. Troiano \& M. Elias, “University access and after: explaining the social composition of degree programmes and the contrasting expectations of students, ” 2013, Higher Education, 67 (5), pp. 637 654. http://doi. org/10. 1007/s10734-013-9670-4.

[16] T. P. Gerber \& S. Y. Cheung, “Horizontal Stratification in Postsecondary Education: Forms, Explanations, and Implications, ” 2008, Annual Review of Sociology, 34(1), pp. $299-318$.

[17] J. Carabaña, “Educación y movilidad social” in V. Navarro (Ed), el Estado del Bienestar en España, 2004, Madrid: Taurus.
[18] J. Carabaña \& G. de la Fuente, “Facultad por Facultad. Origen familiar y empleo de los licenciados en CCSS y Humanidades de la UCM en el año 2003, ” 2016 Revista Complutense de Educación. Vol. 27, 3, pp. 983-1001

[19] S. Fachelli, D. Torrents \& J. Navarro-Cendejas, “iLa universidad española suaviza las diferencias de clase en la inserción laboral?” , 2014, Revista de Educación, pp. $119-144$.

http://ddd. uab. cat/pub/artpub/2014/118532/revedu_a 2014m4-6n364p119iSPA. pdf.

[20] S. Fachelli \& J. Navarro-Cendejas, “Relationship between social original and labor insertion of university graduates, ” 2015, Journal of Educational Research, Assessment and Evaluation RELIEVE, Vol.21, 2, pp. 1-21, https://ddd. uab. cat/pub/artpub/2015/145391/RELIEVE v21n2a1iENG. pdf.

[21] S. Fachelli \& L. Medir, “Determinantes de la decisión de continuar los estudios por parte de graduados universitarios catalanes. Un estudio empírico sobre la influencia de la movilidad geográfica, el origen educativo y el rendimiento académico, ” 2015, Ponencia Grup d' Innovació Docent Consolidat de Gestió i Administració Pública. Universitat de Barcelona.

[22] S. Fachelli \& D. Montolio, Valuation by Catalan graduates of the training received at university in relation to its utility in the workplace, 2015, Mult. J. Edu. Soc \& Tec. Sci. Vol. 2, 2: pp. 14-37. http://polipapers. upv. es/index. php/MUSE.

[23] P. López-Roldán \& S. Fachelli, Metodología de la Investigación Social Cuantitativa. Bellaterra: Dipòsit Digital de Documents, Universitat Auònoma de Barcelona.

http://pagines. uab. cat/plopez/content/manual-misc.

[24] AQU, Universitat i treball a Catalunya 2014. Estudi de la inserció laboral de la població titulada de les universitats catalanes, 2014, Barcelona Agència per a la Qualitat del Sistema Universitari de Catalunya http://www. aqu. cat/doc/doc_14857668_1.pdf. 